\section{Just Noticeable Difference}

Mark Mennemeier

Neurobiology and Developmental Sciences, University of Arkansas for Medical Sciences, Little Rock, AR, USA

\section{Synonyms}

Difference threshold

\section{Definition}

The term just noticeable difference (JND) refers to the amount of change in stimulus intensity necessary to detect a reliable change in sensation. For example, when a stimulus above the absolute threshold is applied to a sense organ, the intensity of the stimulus must be increased by a critical amount (i.e., the JND) before a person is able to report change in sensation.

\section{See Also}

Absolute Threshold

\section{References and Readings}

Gescheider, G. A. (1997). Psychophysics: The fundamentals (3rd ed.). London: Erlbaum, Chapter 1. 